\title{
Article \\ Aorto-Iliac Artery Calcification and Graft Outcomes in Kidney Transplant Recipients
}

\author{
Stan Benjamens ${ }^{1,2}, * \mathbb{0}$, Saleh Z. Alghamdi ${ }^{1}$, Elsaline Rijkse ${ }^{3}$, Charlotte A. te Velde-Keyzer ${ }^{4}$, Stefan P. Berger ${ }^{4}$, \\ Cyril Moers ${ }^{1}{ }^{1}$, Martin H. de Borst $\left.{ }^{4}{ }^{(}\right)$, Riemer H. J. A. Slart ${ }^{2,5}{ }^{(}$, Frank J. M. F. Dor ${ }^{6,7}$, Robert C. Minnee ${ }^{3}$ and \\ Robert A. Pol ${ }^{1} \mathbb{D}$
}

check for

updates

Citation: Benjamens, S.; Alghamdi, S.Z.; Rijkse, E.; te Velde-Keyzer, C.A.; Berger, S.P.; Moers, C.; de Borst, M.H.; Slart, R.H.J.A.; Dor, F.J.M.F.; Minnee, R.C.; et al. Aorto-Iliac Artery Calcification and Graft Outcomes in Kidney Transplant Recipients. J. Clin. Med. 2021, 10, 325. https://doi.org/ $10.3390 /$ jcm10020325

Received: 25 November 2020 Accepted: 14 January 2021 Published: 17 January 2021

Publisher's Note: MDPI stays neutral with regard to jurisdictional clai$\mathrm{ms}$ in published maps and institutional affiliations.

Copyright: (C) 2021 by the authors. Licensee MDPI, Basel, Switzerland. This article is an open access article distributed under the terms and conditions of the Creative Commons Attribution (CC BY) license (https:// creativecommons.org/licenses/by/ $4.0 /)$.
1 Department of Surgery, Division of Transplant Surgery, University of Groningen, University Medical Center Groningen, 9713 GZ Groningen, The Netherlands; s.z.alghamdi@student.rug.nl (S.Z.A.); c.moers@umcg.nl (C.M.); r.pol@umcg.nl (R.A.P.)

2 Medical Imaging Center, Department of Nuclear Medicine and Molecular Imaging, University of Groningen, University Medical Center Groningen, 9713 GZ Groningen, The Netherlands; r.h.j.a.slart@umcg.nl

3 Department of Surgery, Division of HPB and Transplant Surgery, Erasmus MC University Medical Center, 3015 CE Rotterdam, The Netherlands; a.rijkse@erasmusmc.nl (E.R.); r.minnee@erasmusmc.nl (R.C.M.)

4 Department of Internal Medicine, Division of Nephrology, University of Groningen, University Medical Center Groningen, 9713 GZ Groningen, The Netherlands; c.a.keyzer@umcg.nl (C.A.t.V.-K.); s.p.berger@umcg.nl (S.P.B.); m.h.de.borst@umcg.nl (M.H.d.B.)

5 Department of Biomedical Photonic Imaging, Faculty of Science and Technology, University of Twente, 7522 NB Enschede, The Netherlands

6 Imperial College Renal and Transplant Centre, Hammersmith Hospital, Imperial College Healthcare NHS Trust, London W12 0HS, UK; frank.dor@nhs.net

7 Department of Surgery \& Cancer, Imperial College, London SW7 2BU, UK

* Correspondence: s.benjamens@umcg.nl; Tel.: +31-50-361-6161

Abstract: While the association of vascular calcification with inferior patient outcomes in kidney transplant recipients is well-established, the association with graft outcomes has received less attention. With this dual-centre cohort study, we aimed to determine the clinical impact of recipient pre-transplant aorto-iliac calcification, measured on non-contrast enhanced computed tomography (CT)-imaging within three years prior to transplantation (2005-2018). We included 547 patients (61.4\% male, age 60 (interquartile range 51-68) years), with a median follow-up of 3.1 (1.4-5.2) years after transplantation. The aorto-iliac calcification score (CaScore) was inversely associated with one-year estimated-glomerular filtration rate (eGFR) in univariate linear regression analysis (standard $\beta-3.3$ (95\% CI -5.1 to $-1.5, p<0.0001$ ), but not after adjustment for potential confounders, including donor and recipient age $(p=0.077)$. In multivariable Cox regression analyses, a high CaScore was associated with overall graft failure $(p=0.004)$ and death with a functioning graft $(p=0.002)$, but not with death-censored graft failure and graft function decline. This study demonstrated that pre-transplant aorto-iliac calcification is associated with one-year eGFR in univariate, but not in multivariable linear regression analyses. Moreover, this study underlines that transplantation in patients with a high CaScore does not result in earlier transplant function decline or worse death censored graft survival, although ongoing efforts for the prevention of death with a functioning graft remain essential.

Keywords: kidney transplantation; vascular calcification; aorta; iliac artery; graft function; graft failure; graft function decline

\section{Introduction}

Atherosclerosis is an important pathophysiological process responsible for an increased disease burden in kidney transplant candidates and recipients, and is associated with decreased overall survival and increased cardiovascular morbidity and mortality [1-3]. Currently, there is limited published data on the effect of recipient pre-transplant aorto-iliac calcification on kidney graft outcomes. 
Kidney graft outcomes can be divided in short-term outcomes, such as early failure or one-year graft function, and long-term outcomes, such as (death-censored) graft failure and graft function decline [4]. Death-censored graft failure, as return to dialysis or retransplantation, is seen in 10-12.5\% of kidney transplant recipients at 5-6.2 years after transplantation $[5,6]$. Overall graft failure, including death with a functioning graft, is seen at a rate of 5\% each year of follow-up, of which $40-60 \%$ is attributed to death with a functioning graft [7-11]. Graft function decline, as doubling of serum creatinine or graft failure, is seen in $18.8 \%$ of kidney transplant recipients at 5.4 years after transplantation [12]. Short and long-term graft outcomes are strongly associated and both closely linked to patient-specific and transplantation-related risk factors [13-16].

A recent meta-analysis on pre-transplant aorto-iliac calcification found an association with decreased overall survival and overall graft failure. Statistical significance was lacking for the association with death-censored graft failure and graft function. This lack of statistical power with regard to kidney graft outcomes can be attributed to the limited number of studies available, the various methods used for identification of calcification and the small sample sizes of the existing publications [17]. Hence, studies including higher numbers of transplant recipients, focusing on various kidney graft outcomes and applying reliable methods of calcifications assessment, are deemed pivotal.

We previously reported that the aorto-iliac calcification score (CaScore), the adjusted Agatston score, was independently associated with patient outcomes, e.g., risk of early (cardiovascular) death and cardiovascular events [1]. In the present study, we aimed to determine whether the aorto-iliac CaScore, measured as an adjusted Agatston score on pretransplant computed tomography (CT)-imaging, is associated with kidney graft function at one-year after transplantation, death-censored graft failure, overall graft failure (including death), death with a function graft, and graft function decline.

\section{Methods}

\subsection{Study Design}

We performed a dual-centre cohort study in The Netherlands (University Medical Center Groningen (UMCG) and Erasmus University Medical Center (Erasmus MC)), in adult kidney transplant recipients. All patients who underwent screening prior to transplantation by means of a non-contrast enhanced CT between January 2005 and December 2018 were included. CT procedures were performed according to the pre-transplant screening protocol in both transplant centres; selecting patients based on age ( $>50$ years); comorbidities (diabetes mellitus and peripheral artery disease) and dialysis vintage ( $>2$ years). Exclusion criteria were age $<18$ years at the time of transplantation and an interval of more than three years between $\mathrm{CT}$ and transplantation. In the inclusion period, both transplant centres participated in the Eurotranplant Senior Program (ESP), a Eurotransplant allocation scheme matching deceased donors above 65 years-of-age to recipients in a similar age range [18]. The primary outcome measure of this study was estimated-glomerular filtration rate (eGFR) at one-year after transplantation. Secondary outcome measures were death-censored graft failure, defined as return to dialysis or re-transplantation (censoring at time of death), overall graft failure (including death with a functioning graft), death with a functioning graft, and graft function decline, defined as doubling in serum creatinine or graft failure (censoring at time of death). This study was approved by the institutional review board of the UMCG (2017/523) and performed in line with the Declaration of Helsinki and the Declaration of Istanbul on Organ Trafficking and Transplant Tourism.

\subsection{Computed Tomography (CT) Assessment of Aorto-Iliac Calcification}

The applied method for quantifying aorto-iliac calcification, as the Agatston score adjusted for the aorto-iliac trajectory (aorto-iliac CaScore) for non-enhanced CT images, has been described previously [1]. In short, CaScore (syngo.CT CaScoring software, Siemens Healthineers, Erlangen, Germany) was quantified for the abdominal aorta below the origin of the renal arteries, the common iliac artery, and the external iliac artery for the side of the 
transplant anastomosis. The standard calcification threshold of 130 Hounsfield units (HU) was used.

\subsection{Clinical Variables}

Details of this cohort, including baseline cardiovascular risk factors, with history and follow-up of cardiovascular disease, have been published previously [1]. The recipient variables included in the present analyses were gender, age, pre-transplant diabetes mellitus (fasting glucose $\geq 7.0 \mathrm{mmol} / \mathrm{L}$, casual plasma glucose $\geq 11.1 \mathrm{mmol} / \mathrm{L}+$ diabetes symptoms, or glucose tolerance test with 2 -h plasma glucose $\geq 11.1 \mathrm{mmol} / \mathrm{L}$ ), body mass index (BMI in $\mathrm{kg} / \mathrm{m}^{2}$ ), smoking status (non, former, or current), hypercholesterolemia (total cholesterol level $>5.2 \mathrm{mmol} / \mathrm{L}$ or use of lipid lower medication), total cholesterol level $(\mathrm{mmol} / \mathrm{L})$, systolic blood pressure $(\mathrm{mmHg})$, use of antihypertensive medication, dialysis status (pre-emptive, haemodialysis, or peritoneal dialysis), dialysis vintage (months), and number of previous kidney transplantations. The transplant variables included the type of donation (living-donation, donation after circulatory death (DCD), or donation after brain death (DBD)), donor gender and age, number of total human leukocyte antigen (HLA)mismatches, warm ischemia time (minutes), and cold ischemia time (minute). Follow-up variables included eGFR at six-months and one-year, calculated with the Chronic Kidney Disease Epidemiology Collaboration (CKD-EPI) equation $\left(\mathrm{mL} / \mathrm{min}\right.$ per $\left.1.73 \mathrm{~m}^{2}\right)$, laboratory values at one-year after transplantation (serum haemoglobin, calcium, phosphate, albumin, glucose, parathyroid hormone (PTH), and urinary protein excretion), and primary cytomegalovirus (CMV) infection [19]. Short-term outcomes were early graft failure (death-censored), defined as return to dialysis in 30-days after transplantation, delayed graft function, defined as dialysis requirement in the first week after transplantation, acute rejection, defined as biopsy-proven acute rejection (BPAR) or an acute rejection treatment episode without BPAR (non-BPAR, Banff 2015 criteria) [4,20].

\subsection{Statistical Analyses}

For baseline characteristics, patients were stratified into two groups based on the aortoiliac CaScore, being a low CaScore $(0-5600 \mathrm{HU})$ and a high CaScore (>5600 HU). These cut-off values are based on the initial aorto-iliac CaScore analyses, with the high CaScore derived from the highest tertile of CaScores [1]. Data were expressed as mean (standard deviation, SD) for normally distributed variables, as median (interquartile range, IQR) for variables with non-normal distribution, and as number (percentage, \%) for categorical variables. For regression analyses, non-normally distributed variables were transformed to the natural $\log (\ln (x+1))$. Results of linear regression were presented as standardized Beta coefficients (Standard $\beta$ ) and in proportional hazards regression analysis as hazard ratios (HRs), both with $95 \%$ confidence intervals $(95 \% \mathrm{CI})$. The reversed Kaplan-Meier method was used to calculate the median (interquartile range (IQR)) follow-up, considering the date of transplantation as the start of follow-up [21]. In all analyses, a $p$-value $<0.05$ was considered significant.

(Death-censored) graft failure-free and graft function decline-free were calculated using Kaplan-Meier survival curves. The low and high aorto-iliac CaScores were compared for living and deceased-donor kidney transplant recipients with Log-rank testing. Cox proportional hazards regression analysis, for living and deceased-donor kidney transplant recipients, was used to establish the association of the high aorto-iliac CaScore with (deathcensored) graft failure, death with a functioning graft and graft function decline. Linear regression was used to establish the association of the aorto-iliac CaScore (continuous) with one-year eGFR. Models were built with a priori selected covariables. In Model 1, we adjusted for transplant centre (UMCG or Erasmus MC) and time between CT and transplantation. In model 2, we adjusted for model 1 plus donor gender, donor type (living donation, DCD or DBD), cold ischemia time, number of HLA mismatches, recipient gender, diabetes mellitus, smoking, dialysis vintage, number of previous transplantations, statin use pre-transplantation. In model 3, we adjusted for model 2 plus recipient age 
and in model 4 for model 3 plus donor age. For linear regression, laboratory results at one year after transplantation (serum phosphate, serum calcium, serum glucose, serum haemoglobin, serum PTH, proteinuria) and an episode of acute rejection were included as additional variables in model 2. Analysis of interaction terms for the association of the aorto-iliac CaScore (continuous) with one-year eGFR by the covariables recipient age, gender, diabetes, donor age, gender, and donor type, was performed. To quantify the magnitude of confounding by donor age on the association between the aorto-iliac CaScore and one-year eGFR, we used the regression methodology as proposed by Janes et al. [22]. In addition, linear regression was performed after multiple imputation by chained equations (MICE), using predictive mean matching and five imputed datasets, with imputations for serum PTH and proteinuria.

Statistical analyses were performed with R: A Language and Environment for Statistical Computing, version 1.0.153 for Mac (R Foundation for Statistical Computing, Vienna, Austria), using the software R-Packages "MASS", "MICE" "mediation", "survival", "ggplot2", and "survminer".

\section{Results}

\subsection{Characteristics and Short-Term Graft Outcomes}

A total of 547 patients were included, of which 446 in the UMCG and 101 in the Erasmus MC $(61.4 \%$ male, age $60(51,68)$ years). The mean time from CT to transplantation was $0.91(0.72)$ years and the median follow-up was $3.1(1.4,5.2)$ years after transplantation. Figure $\mathrm{S} 1$ shows the numbers for death, graft failure and end of follow-up in the first three years after kidney transplantation. Baseline patient characteristics for the total cohort and CaScore groups are shown in Table 1. In the high compared to the low CaScore group, transplant recipients were older $(66(60,71)$ vs. $55(47,64))$ (Figure S2), patients had a higher donor age (59 (12) vs. 52 (14) years), there were fewer living-donor $(47.3 \%$ vs. $58.9 \%)$, and more DCD ( $29.1 \%$ vs. $20.5 \%)$ and DBD ( $23.6 \%$ vs. $20.5 \%)$ transplantation procedures. In the first year of follow-up, laboratory values $(n=462)$ of the CaScore groups differed for eGFR at six-months and one-year, calcium, phosphate, glucose, PTH, and urinary protein excretion (Table 1). For short-term graft outcomes, more early graft failure (death-censored) events were observed in the high compared to the low CaScore group $(n=9(4.9)$ vs. $n=5$ (1.4), $p=0.027$ ), but no differences in delayed graft function or acute rejections (Table 2). In nine out of the 14 patients with early graft failure, graft failure was classified as due to a vascular cause. In Table S1, patient characteristics are presented for living and deceased donation separately.

Table 1. Characteristics.

\begin{tabular}{|c|c|c|c|c|}
\hline Variables & $\begin{array}{c}\text { Total } \\
(n=547)\end{array}$ & $\begin{array}{l}\text { Low CaScore } \\
\quad(n=365)\end{array}$ & $\begin{array}{l}\text { High CaScore } \\
\qquad(n=182)\end{array}$ & $p$-Value \\
\hline \multicolumn{5}{|l|}{ Patient } \\
\hline Male gender, $n(\%)^{\mathrm{a}}$ & $336(61.4)$ & $205(56.2)$ & $131(72.0)$ & $<0.001^{b}$ \\
\hline Age, years ${ }^{c}$ & $60(51,68)$ & $55(47,64)$ & $66(60,71)$ & $<0.001^{\mathrm{d}}$ \\
\hline Diabetes mellitus, $n(\%)^{\mathrm{a}}$ & $173(31.6)$ & $117(32.1)$ & $56(30.8)$ & $0.836^{\mathrm{b}}$ \\
\hline Body Mass Index, $\mathrm{kg} / \mathrm{m}^{2} \mathrm{~b}$ & $26.8(4.7)$ & $26.9(4.9)$ & $26.7(4.1)$ & $0.670^{\mathrm{c}}$ \\
\hline Smoker, $n(\%)$ a & & & & $0.004^{b}$ \\
\hline Non & $154(28.2)$ & $119(32.6)$ & $35(19.2)$ & \\
\hline Former & $332(60.7)$ & $206(56.4)$ & $126(69.2)$ & \\
\hline Current & $61(11.2)$ & $40(11.0)$ & $21(11.5)$ & \\
\hline Hypercholesterolemia, $n(\%)^{\text {a }}$ & $153(28.0)$ & $96(26.3)$ & $57(31.3)$ & $0.258^{\mathrm{b}}$ \\
\hline Total cholesterol, $\mathrm{mmol} / \mathrm{L}$ & $4.7(1.3)$ & $4.8(1.5)$ & $4.5(1.2)$ & $0.031^{\mathrm{c}}$ \\
\hline Systolic blood pressure, $\mathrm{mmHg}$ & $145(22)$ & $143(21)$ & $148(24)$ & $0.014^{\mathrm{c}}$ \\
\hline Use of antihypertensive medication, $n$ (\%) & $439(80.3)$ & $282(77.3)$ & $157(86.3)$ & $0.017^{\mathrm{b}}$ \\
\hline
\end{tabular}


Table 1. Cont.

\begin{tabular}{|c|c|c|c|c|}
\hline Variables & $\begin{array}{c}\text { Total } \\
(n=547)\end{array}$ & $\begin{array}{l}\text { Low CaScore } \\
\quad(n=365)\end{array}$ & $\begin{array}{l}\text { High CaScore } \\
\quad(n=182)\end{array}$ & $p$-Value \\
\hline Type of dialysis, $n(\%)^{\mathrm{a}}$ & & & & $0.109^{b}$ \\
\hline Pre-emptive & $197(36.0)$ & $142(38.9)$ & $55(30.2)$ & \\
\hline Haemodialysis & $250(45.7)$ & $162(44.4)$ & $88(48.4)$ & \\
\hline Peritoneal dialysis & $100(18.3)$ & $61(16.7)$ & $39(21.4)$ & \\
\hline Dialysis vintage, months ${ }^{c}$ & $12(0,28)$ & $11(0,25)$ & $16(0,33)$ & $0.012^{d}$ \\
\hline Previous transplants, $n(\%)^{\mathrm{a}}$ & & & & $0.872^{b}$ \\
\hline Non & $514(94.0)$ & $343(94.0)$ & $171(94.0)$ & \\
\hline One & $22(4.0)$ & $14(3.8)$ & $8(4.4)$ & \\
\hline Two & $11(2.0)$ & $8(2.2)$ & $3(1.6)$ & \\
\hline Aorto-iliac CaScore, $\mathrm{HU}$ & $2994(294,7888)$ & $856(46,2994)$ & $9732(7918,14315)$ & $<0.0011^{d}$ \\
\hline \multicolumn{5}{|l|}{ Transplantation } \\
\hline Type of donation, $n(\%)^{a}$ & & & & $0.025^{b}$ \\
\hline Living-donation & $301(55.0)$ & $215(58.9)$ & $86(47.3)$ & \\
\hline Donation after circulatory death & $128(23.4)$ & $75(20.5)$ & $53(29.1)$ & \\
\hline Donation after brain death & $118(21.6)$ & $75(20.5)$ & $43(23.6)$ & \\
\hline Male gender donor, $\mathrm{n}(\%)$ & $253(46.3)$ & $160(43.8)$ & $93(51.1)$ & $0.130^{b}$ \\
\hline Donor age, years & $54(14)$ & $52(14)$ & $59(12)$ & $<0.001^{\mathrm{c}}$ \\
\hline No. of HLA-mismatches, $n$ & $3.5(1.5)$ & $3.4(1.5)$ & $3.6(1.4)$ & $0.127^{\mathrm{c}}$ \\
\hline Warm ischemia time, minutes & $42(36)$ & $42(44)$ & $41(13)$ & $0.676^{\mathrm{c}}$ \\
\hline Cold ischemia time, minutes & $433(340)$ & $415(343)$ & $470(331)$ & $0.076^{\mathrm{c}}$ \\
\hline \multicolumn{5}{|l|}{ Follow-up e } \\
\hline eGFR at six-months ${ }^{\text {a }}$ & $50(18)$ & $51(19)$ & $46(17)$ & $0.004^{c}$ \\
\hline eGFR at one-year ${ }^{a}$ & $51(21)$ & $53(20)$ & $48(21)$ & $0.007^{c}$ \\
\hline Haemoglobin at one-year, $\mathrm{mmol} / \mathrm{L}$ & $8.3(3.8)$ & $8.3(4.6)$ & $8.2(1.1)$ & $0.811^{\mathrm{c}}$ \\
\hline Calcium at one-year, $\mathrm{mmol} / \mathrm{L}$ & $2.43(0.14)$ & $2.43(0.14)$ & $2.45(0.14)$ & $0.034^{\mathrm{c}}$ \\
\hline Phosphate at one year, $\mathrm{mmol} / \mathrm{L}$ & $0.93(0.21)$ & $0.95(0.21)$ & $0.89(0.19)$ & $0.004^{\mathrm{c}}$ \\
\hline Albumin at one-year, g/L & $43(3)$ & $43(3)$ & $43(3)$ & $0.234^{c}$ \\
\hline Glucose at one-year, $\mathrm{mmol} / \mathrm{L}$ & $5.9(5.1,7.3)$ & $5.7(5.1,7.2)$ & $6.2(5.4,7.6)$ & $0.005^{\mathrm{d}}$ \\
\hline PTH at one-year, $\mathrm{pmol} / \mathrm{L}$ & $10(7,16)$ & $10(7,15)$ & $12(8,16)$ & $0.064^{\mathrm{d}}$ \\
\hline Protein excretion at one-year, g/ $24 \mathrm{~h}$ & $0.2(0.1,0.3)$ & $0.1(0.1,0.2)$ & $0.2(0.1,0.3)$ & $0.002^{d}$ \\
\hline Cytomegalovirus infection, n (\%) & $29(5.3)$ & $17(4.7)$ & $12(6.6)$ & $0.453^{b}$ \\
\hline
\end{tabular}

HU = Hounsfield units; HLA = human leukocyte antigen; eGFR = estimated glomerular filtration rate; PTH = parathyroid hormone; ${ }^{a}$ eGFR - CKD-EPI (Chronic Kidney Disease Epidemiology Collaboration) $\left(\mathrm{mL} / \mathrm{min}\right.$ per $1.73 \mathrm{~m} 20{ }^{\mathrm{b}}{ }^{\mathrm{p}}$-value by chi-square test; ${ }^{\mathrm{c}} p$-value by Student's $t$-test; ${ }^{\mathrm{d}} p$-value by Mann-Whitney U test; ${ }^{\mathrm{e}}$ data available for 462 patients.

Table 2. Short and long-term graft outcomes (numbers, \%).

\begin{tabular}{ccccc}
\hline Outcome Measures & $\begin{array}{c}\text { Total } \\
(\boldsymbol{n}=\mathbf{5 4 7 )}\end{array}$ & $\begin{array}{c}\text { Low CaScore } \\
(\boldsymbol{n}=\mathbf{3 6 5})\end{array}$ & $\begin{array}{c}\text { High CaScore } \\
(\boldsymbol{n}=\mathbf{1 8 2})\end{array}$ & $\boldsymbol{p}$-Value \\
\hline Median follow-up, years & $3.1(1.4,5.2)$ & $3.2(1.5,5.6)$ & $2.7(1.2,4.2)$ \\
$\quad$ Short-term graft outcomes & & & & $9(4.9)$ \\
Early graft failure (death-censored) & $14(2.6)$ & $5(1.4)$ & $55(30.2)$ & 0.027 \\
Delayed graft function & $155(28.3)$ & $100(27.4)$ & $23(12.6)$ & 1.000 \\
Acute rejection (first-year) & $70(12.8)$ & $47(12.9)$ & $23(12.6)$ & 0.734 \\
Long-term graft outcomes & & $41(11.2)$ & $54(29.7)$ & 0.002 \\
Death-censored graft failure & $64(11.7)$ & $64(17.5)$ & $31(17.0)$ & $<0.001$ \\
$\quad$ Overall graft failure & $118(21.6)$ & $23(6.3)$ & $30(16.5)$ & 0.515 \\
Death with a functioning graft & $54(9.9)$ & $51(14.0)$ & & \\
Graft function decline & $81(14.8)$ &
\end{tabular}

Median follow-up after transplantation (interquartile range); number of short-term and long-term graft outcomes (\%); $p$-value by chisquare test. 


\subsection{Aorto-Iliac Calcification Score (CaScore) and One-Year Kidney Transplant Function}

Mean eGFR at one-year after transplantation $(n=462)$ was $51(21)$, with $48(21)$ in the high and 53 (20) in the low CaScore group. The CaScore (continuous) was inversely associated with one-year eGFR, with a standard $\beta-3.5$ (95\% CI -5.3 to $-1.7, p<0.0001)$ when adjusting for transplant centre and time CT to transplantation (model 1). When adjusting for patient, transplantation and follow-up covariables, with the exception of donor age, this inverse association remained significant (model 2, standard $\beta-4.1$ (95\% CI -4.9 to $-2.4, p<0.0001$ ), and model 3 (including recipient age), standard $\beta-2.7$ (95\% CI -4.6 to $-0.7, p=0.008)$ ). This association lost statistical significance after adjustment for donor age (model 4 , standard $\beta-1.7(95 \% \mathrm{CI}-3.6$ to $0.2, p=0.077)$ ) (Table 3$)$. There was a significant interaction for the univariable association of CaScore with one-year eGFR by donor age $(p<0.0001)$, but not for the covariables recipient age, gender, diabetes, donor gender, and donor type $(p>0.05)$. When quantifying the magnitude of confounding, the direct effect of the aorto-iliac CaScore on one-year eGFR was -3.3 (95\% CI -5.1 to -1.5 ) and the effect on the confounder (donor age) was 5.0 (95\% CI 3.8 to 6.2$)$. The direct effect of the confounder on one-year eGFR was -0.6 ( $95 \% \mathrm{CI}-0.7$ to -0.5$)$, with a bootstrapped indirect effect of $-2.9(95 \% \mathrm{CI}-4.0$ to -1.9$)$. Donor age was identified as a confounder of the association between the aorto-iliac CaScore and one-year eGFR, resulting in an $87.2 \%$ of the association (Figure 1). In Table S2, the linear regression for one-year eGFR is presented for the MICE model. In Table S3, the linear regression in presented for living- and deceased donation separately.

Table 3. Multivariable adjusted associations of the CaScore with one-year eGFR.

\begin{tabular}{cccc}
\hline One-Year eGFR & Standard $\beta$ & $\mathbf{9 5 \%}$ CI & $p$-Value \\
\hline Univariate & -3.3 & -5.1 to -1.5 & $<0.0001$ \\
Model 1 & -3.5 & -5.3 to -1.7 & $<0.0001$ \\
Model 2 & -4.1 & -5.9 to -2.4 & $<0.0001$ \\
Model 3 & -2.7 & -4.6 to -0.7 & 0.008 \\
Model 4 & -1.7 & -3.6 to 0.2 & 0.077 \\
\hline
\end{tabular}

Linear regression analysis, data available for 462 patients. Data are presented as hazard ratio and 95\% confidence interval (CI) for the continuous CaScore (natural log transformed). Model 1: adjusted for transplant centre and time between computed tomography and transplantation; model 2: adjusted for model 1 plus donor gender, donor type (living donation, donation after circulatory death (DCD) or donation after brain death (DBD)), cold ischemia time, no. of human leukocyte antigen (HLA) mismatches, recipient gender, diabetes mellitus, smoking, dialysis vintage, no. of previous transplantations, statin use, laboratory results at one year after transplantation (serum phosphate, serum calcium, serum glucose, serum haemoglobin, serum PTH, proteinuria), and episode of acute rejection; model 3: adjusted for model 2 plus recipient age; model 4: adjusted for model 3 plus donor ag.

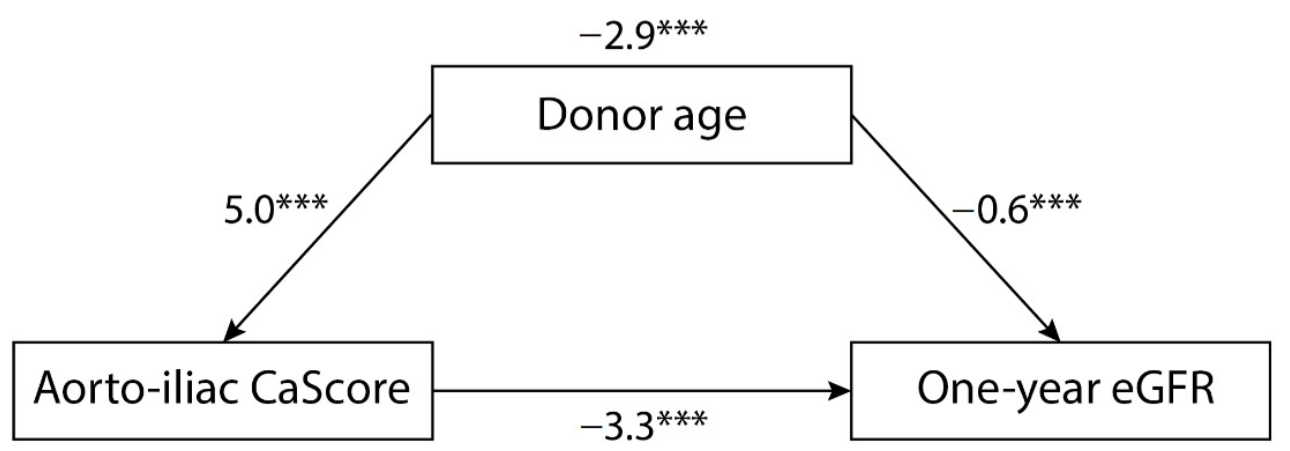

Figure 1. Graphical illustration of the magnitude of confounding for one-year estimated glomerular filtration rate (eGFR), showing the regression coefficients for the associations ${ }^{* * *}$ indicating a $p$-value $<0.0001)$. The direct effect of the aorto-iliac CaScore on one-year eGFR was -3.3 (95\%CI -5.1 to -1.5 ) and the effect on the confounder (donor age) was 5.0 (95\%CI 3.8 to 6.2). The direct effect of the confounder on one-year eGFR was $-0.6(95 \% \mathrm{CI}-0.7$ to -0.5$)$, with a bootstrapped indirect effect of $-2.9(95 \% \mathrm{CI}-4.0$ to -1.9$)$. 


\subsection{Aorto-Iliac CaScore and Death-Censored Graft Failure}

A total of 64 (11.7\%) events of death-censored graft failure occurred, with $23(12.6 \%)$ in the high and $41(11.2 \%)$ in the low CaScore group (Table 2). Kaplan-Meier survival analysis for death-censored graft failure, with the low and high CaScore groups stratified by living and deceased donor kidney transplantation, showed earlier graft failure for the deceased donor-high CaScore group $(p<0.001)$ (Figure 2). Kaplan-Meier survival analysis stratified by transplant recipient age, below or above 65 years-of-age, did not show significant differences ( $p=0.078$, Figure S3). In Cox regression analysis, no significant associations were observed for a high CaScore and death-censored graft failure (model 4, HR 1.1 (95\% CI 0.6 to 2.0, $p=0.711)$ ) (Table 4). No differences were observed when stratifying for living- and deceased donation (model 4, Table S4).

\section{A}
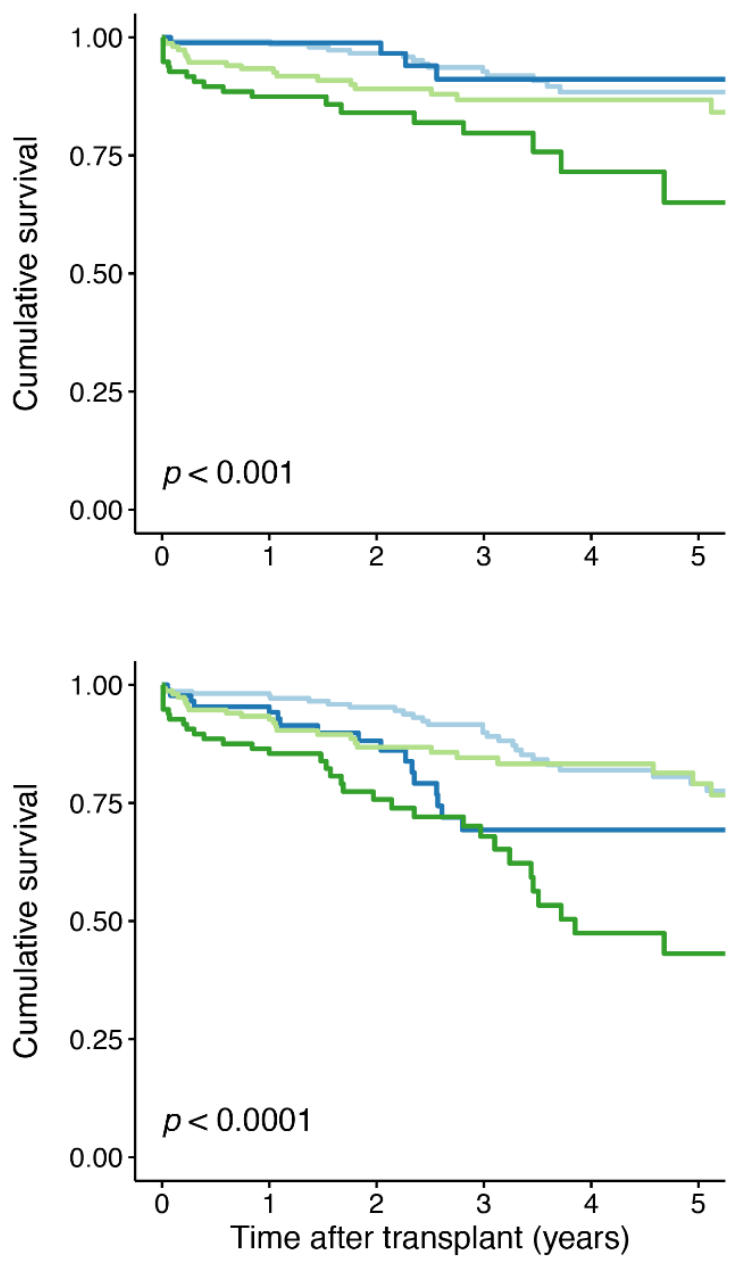

No. at risk

$\begin{array}{rcccccc}\text { Living - low CaScore } & 215 & 211 & 143 & 106 & 67 & 54 \\ \text { Living - high CaScore } & 86 & 82 & 48 & 24 & 16 & 10 \\ \text { Deceased - low CaScore } & 150 & 140 & 93 & 68 & 50 & 35 \\ \text { Deceased - high CaScore } & 96 & 83 & 45 & 30 & 15 & 10\end{array}$

Figure 2. Kaplan-Meier survival curve for (A) death-censored and (B) overall graft failure freesurvival for the low and high aorto-iliac CaScore group, stratified by living and deceased donor kidney transplantation and including a life table applicable for both graphs. Number at risk (No. at risk) provided for all four groups. 
Table 4. Multivariable adjusted associations of the CaScore with (death-censored) graft failure, death with a functioning graft and graft function decline.

\begin{tabular}{|c|c|c|c|c|}
\hline \multirow[t]{2}{*}{ Outcomes Measures } & \multirow{2}{*}{$\begin{array}{l}\text { Low CaScore } \\
\text { Hazard Ratio }\end{array}$} & \multicolumn{3}{|c|}{ High CaScore } \\
\hline & & Hazard Ratio & $95 \%$ CI & $p$-Value \\
\hline \multicolumn{5}{|c|}{ Death censored graft failure } \\
\hline Univariate & 1.0 (Ref) & 1.5 & 0.9 to 2.5 & 0.125 \\
\hline Model 1 & 1.0 (Ref) & 1.6 & 0.9 to 2.7 & 0.082 \\
\hline Model 2 & 1.0 (Ref) & 1.4 & 0.8 to 2.4 & 0.211 \\
\hline Model 3 & 1.0 (Ref) & 1.1 & 0.6 to 2.0 & 0.697 \\
\hline Model 4 & 1.0 (Ref) & 1.1 & 0.6 to 2.0 & 0.711 \\
\hline \multicolumn{5}{|l|}{ Overall graft failure } \\
\hline Univariate & 1.0 (Ref) & 2.4 & 1.7 to 3.5 & $<0.0001$ \\
\hline Model 1 & 1.0 (Ref) & 2.5 & 1.7 to 3.7 & $<0.0001$ \\
\hline Model 2 & 1.0 (Ref) & 2.4 & 1.6 to 3.5 & $<0.0001$ \\
\hline Model 3 & 1.0 (Ref) & 1.8 & 1.2 to 2.7 & 0.008 \\
\hline Model 4 & 1.0 (Ref) & 1.9 & 1.2 to 2.9 & 0.006 \\
\hline \multicolumn{5}{|c|}{ Death with a functioning graft } \\
\hline Univariate & 1.0 (Ref) & 3.8 & 2.2 to 6.5 & $<0.0001$ \\
\hline Model 1 & 1.0 (Ref) & 3.9 & 2.3 to 6.9 & $<0.0001$ \\
\hline Model 2 & 1.0 (Ref) & 4.0 & 2.3 to 7.1 & $<0.0001$ \\
\hline Model 3 & 1.0 (Ref) & 2.8 & 1.5 to 5.2 & 0.002 \\
\hline Model 4 & 1.0 (Ref) & 2.7 & 1.4 to 5.0 & 0.004 \\
\hline \multicolumn{5}{|l|}{ Graft function decline } \\
\hline Univariate & 1.0 (Ref) & 1.6 & 1.0 to 2.6 & 0.038 \\
\hline Model 1 & 1.0 (Ref) & 1.8 & 1.1 to 2.8 & 0.018 \\
\hline Model 2 & 1.0 (Ref) & 1.6 & 1.0 to 2.6 & 0.043 \\
\hline Model 3 & 1.0 (Ref) & 1.3 & 0.8 to 2.1 & 0.368 \\
\hline Model 4 & 1.0 (Ref) & 1.4 & 0.8 to 2.4 & 0.223 \\
\hline
\end{tabular}

Cox proportional hazards regression analysis. Data are presented as hazard ratio and $95 \%$ confidence interval (CI) for the two aorto-iliac CaScore groups (low and high). Model 1: adjusted for transplant centre and time between computed tomography and transplantation; model 2: adjusted for model 1 plus donor gender, donor type (living donation, donation after circulatory death (DCD), cold ischemia time, no. of human leukocyte antigen (HLA) mismatches, recipient gender, diabetes mellitus, dialysis vintage, and no. of previous transplantations, statin use; model 3: adjusted for model 2 plus recipient age; model 4: adjusted for model 3 plus donor age.

\subsection{Aorto-Iliac CaScore and Overall Graft Failure}

A total of 118 (21.6\%) events of overall graft failure occurred, with $54(29.7 \%)$ in the high and $64(17.5 \%)$ in the low CaScore group (Table 2). Kaplan-Meier survival analysis for overall graft failure, with the low and high CaScore group stratified by living and deceased donor kidney transplantation, showed earlier graft failure for the living and deceased donor-high CaScore groups $(p<0.0001)$ (Figure 2). Kaplan-Meier survival analysis stratified by transplant recipient age showed, a lower survival for the recipients $\geq 65$ years-of-age - high CaScore group $(p<0.0001)$ (Figure S3). In Cox regression analysis, a significant association was observed for a high CaScore and graft failure after adjusting for patient and transplantation covariables, including recipient and donor age (model 4, HR $1.9(95 \%$ CI 1.2 to $2.9, p=0.006)$ ) (Table 4$)$. When stratifying for donor status, the association remained significant for deceased donation (model $4, p=0.048$ ) and for living-donation (model $4, p=0.0036$ ) (Table S3).

\subsection{Aorto-Iliac CaScore and Death with a Functioning Graft}

A total of $54(9.9 \%)$ events of death with a functioning graft occurred, with $31(17.0 \%)$ in the high and $23(6.3 \%)$ in the low CaScore group (Table 2). Kaplan-Meier survival analysis for death with a functioning graft, with the low and high CaScore group stratified by living and deceased donor kidney transplantation, showed earlier graft failure for the living and deceased donor-high CaScore groups $(p<0.0001)$ (Figure 3$)$. Kaplan-Meier survival analysis stratified by transplant recipient age showed, a lower survival for the recipients $\geq 65$ years-of-age- - high CaScore group $(p<0.0001)$ (Figure S4). In Cox regression analysis, a significant association was observed for a high CaScore and death with a functioning 
graft after adjusting for patient and transplantation covariables, including recipient and donor age (model 4, HR 2.7 (95\% CI 1.4 to 5.0, $p=0.224$ )) (Table 4). When stratifying for donor status, the association remained significant for both living- and deceased-donation (model $4, p=0.015$ and $p=0.042$, respectively) (Table S4).

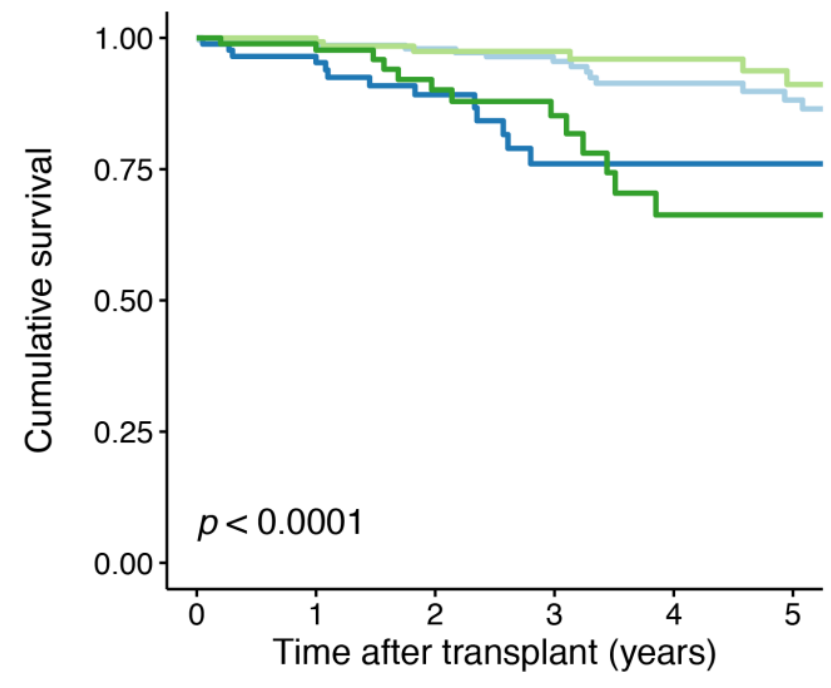

No. at risk

$\begin{array}{rcccccc}\text { Living-low CaScore } & 215 & 211 & 143 & 106 & 67 & 54 \\ \text { Living-high CaScore } & 86 & 82 & 48 & 24 & 16 & 10 \\ \text { Deceased-low CaScore } & 150 & 140 & 93 & 68 & 50 & 35 \\ \text { Deceased-high CaScore } & 96 & 83 & 45 & 30 & 15 & 10\end{array}$

Figure 3. Kaplan-Meier survival curve for death with a functioning graft free-survival for the low and high aorto-iliac CaScore group, stratified by living and deceased donor kidney transplantation. Number at risk (No. at risk) provided for all four groups.

\subsection{Aorto-Iliac CaScore and Graft Function Decline}

A total of $81(14.8 \%)$ events of graft function decline occurred, with $51(14.0 \%)$ in the high and $30(16.5 \%)$ in the low CaScore group (Table 2). Kaplan-Meier survival analysis for graft function decline, with the low and high CaScore group stratified by living and deceased donor kidney transplantation, showed a lower time-to-graft function decline for deceased donor-high CaScore group $(p<0.0001)$ (Figure 4). Kaplan-Meier survival analysis stratified by transplant recipient age, showed a lower survival for the recipients $\geq 65$ years-of-age - high CaScore group $(p=0.014)$ (Figure S5). In Cox regression analysis, no significant associations were observed between the high CaScore and graft function decline after adjusting for patient and transplantation covariables, including recipient and donor age (model 4, HR 1.4 (95\% CI 0.8 to 2.4, $p=0.223$ ) (Table 4). No differences were observed when stratifying for living- and deceased donation (model 4, Table S4). 


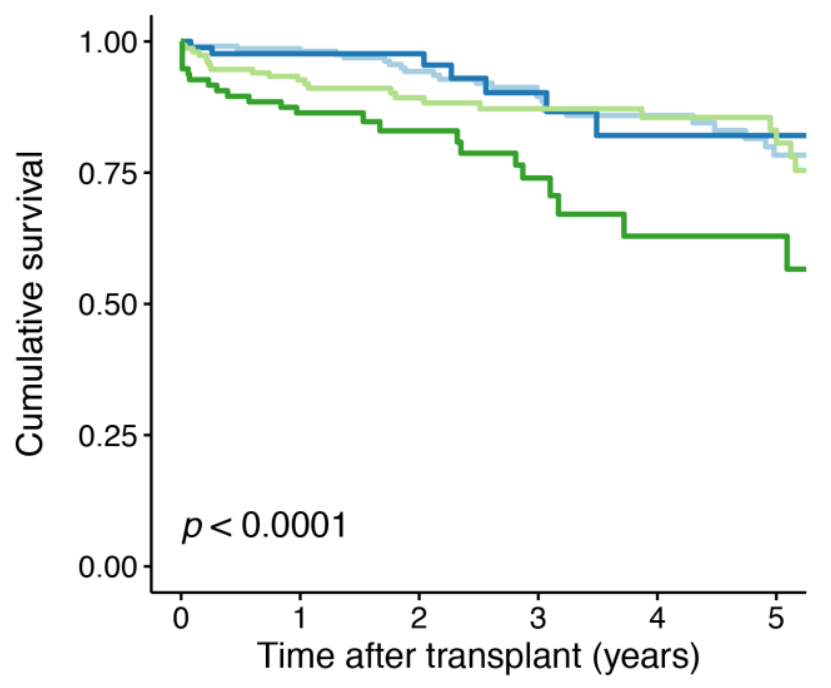

No. at risk

$\begin{array}{rcccccc}\text { Living - low CaScore } & 215 & 210 & 139 & 103 & 67 & 49 \\ \text { Living - high CaScore } & 86 & 80 & 49 & 25 & 15 & 9 \\ \text { Deceased - low CaScore } & 150 & 138 & 93 & 68 & 49 & 34 \\ \text { Deceased - high CaScore } & 96 & 81 & 44 & 27 & 14 & 10\end{array}$

Figure 4. Kaplan-Meier survival curve for graft function decline free-survival for the low and high aorto-iliac CaScore group, stratified by living and deceased donor kidney transplantation. Number at risk (No. at risk) provided for all four groups.

\section{Discussion}

In this dual centre cohort study, we identified a non-causal inverse association between pre-transplant aorto-iliac calcification and one-year eGFR in univariate analysis. This association was not significant after adjustment for patient-specific, transplantation-related, and follow-up covariables, including donor and recipient age. The increased donor age in older recipients, due to the allocation of older donor kidneys to older recipients in the Eurotransplant Senior Program, was a significant confounder. For long-term graft outcomes, no association could be established between a high aorto-iliac CaScore and an increased risk of death-censored graft failure or graft function decline, in both living and deceased donor kidney transplantation. Whereas the identified association between a high aorto-iliac CaScore and graft failure (death-censored) remained significant after adjustment for various covariables, including donor and recipient age. Also, the high aorto-iliac CaScore was associated with death with a functioning graft.

Our study has both similarities and important differences compared to four previously published studies on pre-transplant imaging-based visualization of calcification. In the largest study $(n=374)$, aorto-iliac stenosis on CT-imaging was not an independent risk factor for graft failure, but was associated with an increased risk of overall graft failure [23]. In a relatively small study $(n=119)$ with lateral lumbar radiography-based calcification assessment, transplant recipients with and without calcifications differed with regard to graft failure-free survival [24]. In a study on pre-transplant CT-based quantification of calcification $(n=100)$, no statistically significant difference in eGFR at one-year after transplantation was found for the quartiles of aorto-iliac calcification. Also, survival analysis for graft failure, with nine events after a median follow-up of 2.6 years, did not show differences between the quartiles [25]. In a cohort of 93 transplant recipients, Aitken et al. showed that visual two group stratification of vascular calcification on pelvic X-ray did result in graft survival differences, but not in differences for one-year creatinine [26]. Compared to these imaging studies, the current study has the largest sample size and a relatively long period of follow-up. The results of our study were comparable for living- 
and deceased-donation, with an exception for the outcome measure graft function decline. In univariate analyses, there was a significant association between the CaScore and graft function decline for deceased-donation, whereas the association was not significant for living-donation. A possible explanation is the significant age difference and difference in diabetes mellitus prevalence between the living-and deceased-donation patients.

The outcomes of our study are in line with previous non-imaging studies on calcification. In a histology-based cohort study $(n=90)$, differences in graft failure were observed for transplant recipients with and without intimal microcalcification of the iliac artery; however, these differences were not tested in multivariable analyses. For graft function decline, an independent association with the combination of intimal microcalcification with positive intimal fetuin-A was identified [27]. In post-transplant blood serum analysis ( $n=699)$, calciprotein particle maturation time (T50), a measure for blood calcification propensity, were shown to be associated with all-cause mortality, cardiovascular mortality, and graft failure [28].

A global development in transplant programs is a steady increase in donor and transplant candidate age [29]. With this trend, the causal relation of recipient age with vascular calcification and the confounding effect of donor age on the association between calcification and outcomes, can be important factors affecting transplant outcomes. Efforts could go to the optimization of kidney function and patient survival for transplant recipients with a high degree of aorto-iliac calcification. The kidney function of grafts from older donors may be improved, by decreasing cold ischemia time, reducing kidney rewarming during transplantation, and optimizing the use of hypothermic and normothermic machine perfusion [30-33]. To improve patient survival in general and death with a functioning graft specially, focus might go to the reduction of modifiable risk factors for cardiovascular disease in kidney transplant candidates and recipients. This risk reduction, pre- and post-transplantation, could be achieved by focusing on prevention of new-onset diabetes, preventive measures for mineral and bone disorders, and reduction of hyperlipidaemia and hypertension [34]. Medical strategies in line with these focus points are age-adapted immunosuppressive therapy, stringent use of statins and antihypertensive medication, and general lifestyle interventions [35-37].

Our study has a few limitations that need to be addressed. First, we retrospectively selected patients in which a CT procedure was already performed based on predefined criteria, making the study prone to selection bias and confounding. Although the presented findings are applicable to the majority of transplant recipients with comparable baseline characteristics (median age of 60 (51-68) years), they should not be extrapolated to relatively young patients with limited comorbidities. Second, the two transplant centres in this cohort study are part of Eurotransplant Senior Program [18,38]. This particular allocation scheme could have influenced the association between the aorto-iliac CaScore and donor age, given the causal relationship between vascular calcification and recipient age. Third, the period of follow-up of 3.1 years (median) is relatively long compared to previous imaging studies on vascular calcification but on the short side compared to large registry studies on the topic of kidney graft outcomes. Fourth, for a relatively large proportion of patients $(n=85$; of which 31 patients experienced overall graft failure with the first year) laboratory values at one-year after transplantation were not available. Complying with general standards for dealing with missing data, a complete case analysis was found to be most reliable. In addition, linear regression was performed after multiple imputation, with imputations for serum PTH and proteinuria. Fifth, the interpretation of the implications of a statistically significant difference should always include a reflection of the clinical significance of the observed difference. In this study, a mean difference in eGFR of $5 \mathrm{~mL} / \mathrm{min}$ per $1.73 \mathrm{~m}^{2}$ was found between a high and a low aorto-iliac CaScore (mean 48 (21) vs. 53 (20)). With a mean eGFR of 51 in the total cohort, this is a 10\% difference in one-year graft function.

Nonetheless, this study has its unique strengths, which justify attention. This is the first study evaluating the association between aorto-iliac calcification and one-year graft function in multivariable analysis, including patient, transplantation, and follow-up 
covariables. Second, the aorto-iliac CaScore is an easy-to-implement assessment, as the software is readily available in standard imaging analysis packages and pre-transplant CT-imaging is advised for high-risk transplant candidates [39,40]. Third, the discrepancy between the results of Kaplan-Meier survival analysis and multivariable Cox regression analyses highlights the importance of relatively large cohort studies that enable adjustment for important covariables. Kaplan-Meier survival analysis indicated significant differences based on the aorto-iliac CaScore for (death-censored) graft failure and graft function decline, whereas a significant independent association in Cox regression analysis was only found for overall graft failure and death with a function graft.

\section{Conclusions}

In conclusion, this study demonstrated that pre-transplant aorto-iliac calcification is associated with one-year eGFR in univariate, but not in multivariable linear regression analyses. The increased donor age in older recipients is a significant confounder for this association. A high CaScore was not associated with death-censored graft failure and graft function decline, whereas independent associations with overall graft failure and death with a functioning graft were identified. These results underline that transplantation in patients with a high CaScore does not result in earlier transplant function decline or worse death censored graft survival, although ongoing efforts for the prevention of death with a functioning graft remain essential.

Supplementary Materials: The following are available online at https://www.mdpi.com/2077-038 $3 / 10 / 2 / 325 / s 1$, Figure S1. Flowchart for death, graft failure and end of follow-up in the first three years after kidney transplantation; Figure S2. Distribution of patients with a high CaScore for eight age groups; Figure S3. Kaplan-Meier survival curve for graft failure and graft loss free-survival for the low and high aorto-iliac CaScore group, stratified by transplant recipient age ( $<65$ or $\geq 65$ years of age), including a life table applicable for both graphs; Figure S4. Kaplan-Meier survival curve for death with a functioning graft free-survival for the low and high aorto-iliac CaScore group stratified by transplant recipient age ( $<65$ or $\geq 65$ years of age); Figure S5. Kaplan-Meier survival curve for graft function decline free-survival for the low and high aorto-iliac CaScore group stratified by transplant recipient age ( $<65$ or $\geq 65$ years of age); Table S1. Characteristics stratified by donor status; Table S2. Multivariable adjusted associations of the CaScore with one-year eGFR after multiple imputation by chained equations; Table S3. Multivariable adjusted associations of the CaScore with one-year eGFR; Table S4. Multivariable adjusted associations of the CaScore with (death-censored) graft failure, death with a functioning graft and graft function decline.

Author Contributions: Conceptualization, S.B. and R.A.P.; methodology, S.B. and R.A.P.; formal analysis, S.B., S.Z.A., E.R., and C.A.t.V.-K.; investigation, S.P.B., C.M., M.H.d.B., R.H.J.A.S., F.J.M.F.D., R.C.M., and R.A.P.; data curation, S.B., S.Z.A. and E.R.; writing-original draft, S.B., S.Z.A., E.R., and C.A.t.V.-K.; writing-review and editing, S.P.B., C.M., M.H.d.B., R.H.J.A.S., F.J.M.F.D., R.C.M., and R.A.P.; visualization, S.B. and S.Z.A.; supervision, R.A.P., R.H.J.A.S., F.J.M.F.D., and R.C.M.; project administration, S.B., S.Z.A. and E.R.; funding acquisition, S.B. and R.A.P. All authors have read and agreed to the published version of the manuscript.

Funding: This work was supported by the Ubbo Emmius Fund (Ubbo Emmius Fund-Junior Scientific Masterclass (UEF-JSM) Talent Grant).

Institutional Review Board Statement: The study was conducted according to the guidelines of the Declaration of Helsinki, and approved by the Institutional Review Board of the University Medical Center Groningen (2017/523).

Informed Consent Statement: Patient consent was waived due to the retrospective study design and full anonymity of the included patients.

Data Availability Statement: The data presented in this study are available on request from the corresponding author. The data are not publicly available due to privacy regulations. 
Acknowledgments: This research project is supported by a grant from the Ubbo Emmius Fund (Ubbo Emmius Fund-Junior Scientific Masterclass (UEF-JSM) Talent Grant). We acknowledge Susanna M.M. Bruins and Tim D.A. Swaab for their assistance with the data collection.

Conflicts of Interest: The authors declare no conflict of interest. The funder had no role in the design of the study; in the collection, analyses, or interpretation of data; in the writing of the manuscript; or in the decision to publish the results.

\section{References}

1. Benjamens, S.; Rijkse, E.; te Velde-Keyzer, C.A.; Berger, S.P.; Moers, C.; de Borst, M.H.; Yakar, D.; Slart, R.H.J.A.; Dor, F.J.M.F.; Minnee, R.C.; et al. Aorto-iliac artery calcification prior to kidney transplantation. J. Clin. Med. 2020, 9, 2893. [CrossRef] [PubMed]

2. Rangaswami, J.; Mathew, R.O.; Parasuraman, R.; Tantisattamo, E.; Lubetzky, M.; Rao, S.; Yaqub, M.S.; Birdwell, K.A.; Bennett, W.; Dalal, P.; et al. Cardiovascular disease in the kidney transplant recipient: Epidemiology, diagnosis and management strategies. Nephrol. Dial. Transpl. 2019, 34, 760-773. [CrossRef] [PubMed]

3. Cianciolo, G.; Capelli, I.; Angelini, M.L.; Valentini, C.; Baraldi, O.; Scolari, M.P.; Stefoni, S. Importance of vascular calcification in kidney transplant recipients. Am. J. Nephrol. 2014, 39, 418-426. [CrossRef] [PubMed]

4. Hamed, M.O.; Chen, Y.; Pasea, L.; Watson, C.J.; Torpey, N.; Bradley, J.A.; Pettigrew, G.; Saeb-Parsy, K. Early graft loss after kidney transplantation: Risk factors and consequences. Am. J. Transpl. 2015, 15, 1632-1643. [CrossRef] [PubMed]

5. De Vusser, K.; Lerut, E.; Kuypers, D.; Vanrenterghem, Y.; Jochmans, I.; Monbaliu, D.; Pirenne, J.; Naesens, M. The predictive value of kidney allograft baseline biopsies for long-term graft survival. J. Am. Soc. Nephrol. 2013, 24, 1913-1923. [CrossRef] [PubMed]

6. $\quad$ Issa, N.; Lopez, C.L.; Denic, A.; Taler, S.J.; Larson, J.J.; Kremers, W.K.; Ricaurte, L.; Merzkani, M.A.; Alexander, M.P.; Chakkera, H.A.; et al. Kidney structural features from living donors predict graft failure in the recipient. J. Am. Soc. Nephrol. 2020, 31, 415-423. [CrossRef] [PubMed]

7. Burton, H.; Iyamu Perisanidou, L.; Steenkamp, R.; Evans, R.; Mumford, L.; Evans, K.M.; Caskey, F.J.; Hilton, R. Causes of renal allograft failure in the UK: Trends in uk renal registry and national health service blood and transplant data from 2000 to 2013. Nephrol. Dial. Transpl. 2019, 34, 355-364. [CrossRef]

8. Naesens, M.; Kuypers, D.R.J.; de Vusser, K.; Evenepoel, P.; Claes, K.; Bammens, B.; Meijers, B.; Sprangers, B.; Pirenne, J.; Monbaliu, D.; et al. The histology of kidney transplant failure: A long-term follow-up study. Transplantation 2014, 98, 427-435. [CrossRef]

9. Van Loon, E.; Senev, A.; Lerut, E.; Coemans, M.; Callemeyn, J.; Van Keer, J.M.; Daniëls, L.; Kuypers, D.; Sprangers, B.; Emonds, M.P.; et al. Assessing the complex causes of kidney allograft loss. Transplantation 2020, 104. [CrossRef]

10. Van Loon, E.; Bernards, J.; van Craenenbroeck, A.H.; Naesens, M. The causes of kidney allograft failure: More than alloimmunity. Transplantation 2020, 104, e46-e56. [CrossRef]

11. Lamb, K.E.; Lodhi, S.; Meier-Kriesche, H.-U. Long-term renal allograft survival in the United States: A critical reappraisal. Am. J. Transpl. 2011, 11, 450-462. [CrossRef] [PubMed]

12. Sotomayor, C.G.; Gomes-Neto, A.W.; Eisenga, M.F.; Nolte, I.M.; Anderson, J.L.; de Borst, M.H.; Osté, M.C.; Rodrigo, R.; Gans, R.O.; Berger, S.P.; et al. Consumption of fruits and vegetables and cardiovascular mortality in renal transplant recipients: A prospective cohort study. Nephrol. Dial. Transpl. 2020, 35, 357-365. [CrossRef] [PubMed]

13. Schnitzler, M.A.; Lentine, K.L.; Gheorghian, A.; Axelrod, D.; Trivedi, D.; L'Italien, G. Renal function following living, standard criteria deceased and expanded criteria deceased donor kidney transplantation: Impact on graft failure and death. Transpl. Int. 2012, 25, 179-191. [CrossRef] [PubMed]

14. Opelz, G.; Döhler, B. Influence of time of rejection on long-term graft survival in renal transplantation. Transplantation 2008, 85, 661-666. [CrossRef] [PubMed]

15. Gaston, R.S.; Fieberg, A.; Hunsicker, L.; Kasiske, B.L.; Leduc, R.; Cosio, F.G.; Gourishankar, S.; Grande, J.; Mannon, R.B.; Rush, D.; et al. Late graft failure after kidney transplantation as the consequence of late versus early events. Am. J. Transpl. 2018, 18, 1158-1167. [CrossRef] [PubMed]

16. Kasiske, B.L.; Israni, A.K.; Snyder, J.J.; Skeans, M.A. Patient outcomes in renal transplantation (port) investigators the relationship between kidney function and long-term graft survival after kidney transplant. Am. J. Kidney Dis. 2011, 57, 466-475. [CrossRef] [PubMed]

17. Rijkse, E.; Dam, J.L.; Roodnat, J.I.; Kimenai, H.J.A.N.; IJzermans, J.N.M.; Minnee, R.C. The prognosis of kidney transplant recipients with aorto-iliac calcification: A systematic review and meta-analysis. Transpl. Int. 2020, 33, 483-496. [CrossRef]

18. Schachtner, T.; Otto, N.M.; Reinke, P. Two decades of the Eurotransplant Senior Program: The gender gap in mortality impacts patient survival after kidney transplantation. Clin. Kidney J. 2020, 13, 1091-1100. [CrossRef]

19. Levey, A.S.; Stevens, L.A.; Schmid, C.H.; Zhang, Y.L.; Castro, A.F.; Feldman, H.I.; Kusek, J.W.; Eggers, P.; Van Lente, F.; Greene, T.; et al. A new equation to estimate glomerular filtration rate. Ann. Intern. Med. 2009, 150, 604-612. [CrossRef]

20. Loupy, A.; Haas, M.; Solez, K.; Racusen, L.; Glotz, D.; Seron, D.; Nankivell, B.J.; Colvin, R.B.; Afrouzian, M.; Akalin, E.; et al. The banff 2015 kidney meeting report: Current challenges in rejection classification and prospects for adopting molecular pathology. Am. J. Transpl. 2017, 17, 28-41. [CrossRef]

21. Clark, T.G.; Bradburn, M.J.; Love, S.B.; Altman, D.G. Survival analysis Part I: Basic concepts and first analyses. Br. J. Cancer 2003, 89, 232-238. [CrossRef] [PubMed] 
22. Janes, H.; Dominici, F.; Zeger, S. On quantifying the magnitude of confounding. Biostatistics 2010, 11, 572-582. [CrossRef] [PubMed]

23. Rijkse, E.; Kimenai, H.J.A.N.; Roodnat, J.I.; Ten Raa, S.; Bijdevaate, D.C.; van Dam, J.L.; Muller, K.; IJzermans, J.N.M.; van der Zijden, M.A.; Minnee, R.C. Impact of aortoiliac stenosis on graft and patient survival in kidney transplant recipients using the tasc ii classification. Transplantation 2019, 103, 2164-2172. [CrossRef] [PubMed]

24. Munguia, P.; Caramelo, R.; Rubio, M.V.; Sahdalá, L.; Arnaudas, L.; Paul, J.; Blasco, Á.; Lou, L.M.; Aladren, M.J.; Sanjuan, A.; et al. Pre-transplant assessment of vascular calcification as a risk factor of mortality, graft loss, and cardiovascular events in renal transplant recipients. Transpl. Proc. 2015, 47, 2368-2370. [CrossRef] [PubMed]

25. Chavent, B.; Maillard, N.; Boutet, C.; Albertini, J.-N.; Duprey, A.; Favre, J.-P. Prognostic value of aortoiliac calcification score in kidney transplantation recipients. Ann. Vasc. Surg. 2017, 44, 245-252. [CrossRef]

26. Aitken, E.; Ramjug, S.; Buist, L.; Kingsmore, D. The Prognostic Significance of Iliac Vessel Calcification in Renal Transplantation. In Transplantation Proceedings; Elsevier: Amsterdam, The Netherlands, 2012.

27. Hwang, H.S.; Lim, S.W.; Sun, I.O.; Yang, K.S.; Yoon, H.E.; Chung, B.H.; Choi, B.S.; Choi, Y.J.; Kim, J., II; Sung Moon, I.; et al. Clinical significance of preexisting microcalcification in the iliac artery in renal transplant recipients. Transplantation 2015, 99, 811-817. [CrossRef]

28. Keyzer, C.A.; de Borst, M.H.; van den Berg, E.; Jahnen-Dechent, W.; Arampatzis, S.; Farese, S.; Bergmann, I.P.; Floege, J.; Navis, G.; Bakker, S.J.L.; et al. Calcification propensity and survival among renal transplant recipients. J. Am. Soc. Nephrol. 2016, 27, 239-248. [CrossRef]

29. Veroux, M.; Grosso, G.; Corona, D.; Mistretta, A.; Giaquinta, A.; Giuffrida, G.; Sinagra, N.; Veroux, P. Age is an important predictor of kidney transplantation outcome. Nephrol. Dial. Transpl. 2012, 27, 1663-1671. [CrossRef]

30. Benjamens, S.; van den Berg, T.A.J.; Kuipers, T.G.J.; Moers, C.; Berger, S.P.; Leuvenink, H.G.D.; Pol, R.A. Kidney temperature during living donor kidney transplantation is associated with short-term measured glomerular filtration rate-A prospective study. Transpl. Int. 2020, 33, 174-180. [CrossRef]

31. O'Neill, S.; Srinivasa, S.; Callaghan, C.J.; Watson, C.J.; Dark, J.H.; Fisher, A.J.; Wilson, C.H.; Friend, P.J.; Johnson, R.; Forsythe, J.L.; et al. Novel organ perfusion and preservation strategies in transplantation-where are we going in the UK? Transplantation $\mathbf{2 0 2 0}$ 104. [CrossRef]

32. Gill, J.; Dong, J.; Eng, M.; Landsberg, D.; Gill, J.S. Pulsatile perfusion reduces the risk of delayed graft function in deceased donor kidney transplants, irrespective of donor type and cold ischemic time. Transplantation 2014, 97, 668-674. [CrossRef] [PubMed]

33. Peters-Sengers, H.; Houtzager, J.H.E.; Idu, M.M.; Heemskerk, M.B.A.; van Heurn, E.L.W.; van der Heide, J.J.H.; Kers, J.; Berger, S.P.; van Gulik, T.M.; Bemelman, F.J. Impact of cold ischemia time on outcomes of deceased donor kidney transplantation: An Analysis of a national registry. Transpl. Direct 2019, 5, e448. [CrossRef] [PubMed]

34. Jardine, A.G.; Gaston, R.S.; Fellstrom, B.C.; Holdaas, H. Prevention of cardiovascular disease in adult recipients of kidney transplants. Lancet 2011, 378, 1419-1427. [CrossRef]

35. Krenzien, F.; ElKhal, A.; Quante, M.; Cetina Biefer, H.R.; Hirofumi, U.; Gabardi, S.; Tullius, S.G. A rationale for age-adapted immunosuppression in organ transplantation. Transplantation 2015, 99, 2258-2268. [CrossRef]

36. Sotomayor, C.G.; te Velde-Keyzer, C.A.; de Borst, M.H.; Navis, G.J.; Bakker, S.J.L. Lifestyle, inflammation, and vascular calcification in kidney transplant recipients: Perspectives on long-term outcomes. J. Clin. Med. 2020, 9, 1911. [CrossRef] [PubMed]

37. Sotomayor, C.G.; Benjamens, S.; Gomes-Neto, A.W.; Pol, R.A.; Groothof, D.; te Velde-Keyzer, C.A.; Chong, G.; Glaudemans, A.W.J.M.; Berger, S.P.; Bakker, S.J.L.; et al. Bone Mineral Density and Aortic Calcification. Transplantation 2020, 105. [CrossRef]

38. Peters-Sengers, H.; Berger, S.P.; Heemskerk, M.B.A.; Al Arashi, D.; van der Heide, J.J.H.; Hemke, A.C.; Ten Berge, I.J.M.; Idu, M.M.; Betjes, M.G.H.; van Zuilen, A.D.; et al. Stretching the limits of renal transplantation in elderly recipients of grafts from elderly deceased donors. J. Am. Soc. Nephrol. 2017, 28, 621-631. [CrossRef]

39. Benjamens, S.; Glaudemans, A.W.J.M.; Berger, S.P.; Slart, R.H.J.A.; Pol, R.A. Have we forgotten imaging prior to and after kidney transplantation? Eur. Radiol. 2018, 28, 3263-3267. [CrossRef]

40. Chadban, S.J.; Ahn, C.; Axelrod, D.A.; Foster, B.J.; Kasiske, B.L.; Kher, V.; Kumar, D.; Oberbauer, R.; Pascual, J.; Pilmore, H.L.; et al. Summary of the kidney disease: Improving global outcomes (KDIGO) clinical practice guideline on the evaluation and management of candidates for kidney transplantation. Transplantation 2020, 104, 708-714. [CrossRef] 